Check for updates

Cite this: RSC Adv., 2017, 7, 45862

\section{A pair of novel Zn(II) enantiomeric coordination polymers based on a chiral multicarboxylate ligand: synthesis, crystal structures and molecular recognition properties $\dagger$}

\author{
Lei Gu, ${ }^{a}$ Han-Zhong Zhang, ${ }^{a}$ Wen-Hong Jiang, ${ }^{a}$ Guang-Feng Hou, ${ }^{b}$ Ying-Hui Yu (D a \\ and Dong-Sheng $\mathrm{Ma}$ (D) *a
}

A pair of $\mathrm{Zn}(I)$ enantiomeric coordination polymers, 1- $R$ and 1-S, namely, $\left[\mathrm{Zn}_{4}\left((R)-\mathrm{H}_{3} \mathrm{~L}\right)_{2}(\mathrm{OH})_{2}\left(\mathrm{H}_{2} \mathrm{O}\right)_{2}\right] \cdot 3 \mathrm{H}_{2} \mathrm{O}$ $\left\{\mathrm{H}_{3} \mathrm{~L}=(R)\right.$ - or $(S)$-5-(1-carboxyethoxymethyl)-isophthalic acid\}, were synthesized via solvothermal reactions. Their structures were determined by single-crystal X-ray diffraction analysis and further characterized by IR spectra, elemental analyses, thermogravimetric analysis (TGA), solid-state circular dichroism (CD) and powder X-ray diffraction (PXRD). The pair of enantiomers crystallized in the same chiral space group, featuring a 3,6-C net and kgd topological type structure with a point symbol of $\left(4^{3}\right)_{2}\left(4^{6} \cdot 6^{6} \cdot 8^{3}\right)$. Their CD spectra exhibit obvious positive or negative Cotton effects, indicating their homochirality. 1- $R$ and 1-S also proved to be second-harmonic generation (SHG) active. Moreover, their fluorescent selective recognition towards nitro aromatic compounds and metal cations were also investigated.
Received 31st July 2017

Accepted 21st September 2017

DOI: $10.1039 / \mathrm{c} 7 \mathrm{ra0} 8433 a$

rsc.li/rsc-advances
As one of ongoing research in our laboratory, several different chiral carboxylate based ligands have been synthesized and investigated. ${ }^{5}$ Inspired by the aforementioned considerations, we synthesized a pair of chiral tridentate carboxylic acid ligands: $(R)$ - and $(S)$-5-(1-carboxyethoxymethyl)-isophthalic acid $\left(\mathrm{H}_{3} \mathrm{~L}\right.$ ) (Scheme 1). Choosing a pair of enantiomorphic $\mathrm{H}_{3} \mathrm{~L}$ ligands as the organic spacers is mainly based on the following consideration: (a) as a multicarboxylic ligand with one flexible carboxylic group and two rigid carboxylic groups, the multiple bridging modes of carboxylic moieties increased the possibility of the generation of fascinating structures with high dimensions. (b) A pair of enantiomerically pure ligands $\left((\boldsymbol{R})-\mathbf{H}_{3} \mathbf{L}\right.$ and $\left.(S)-\mathbf{H}_{3} \mathbf{L}\right)$ could efficiently ensure the production of a pair of enantiomorphic CCPs, the comparative investigation of whose structures and functional properties might be interesting and meaningful.

Based on the above two enantiomers and zinc as the metal center, a pair of CCPs, namely, $\left[\mathrm{Zn}_{4}\left((R)-\mathrm{H}_{3} \mathrm{~L}\right)_{2}(\mathrm{OH})_{2}\left(\mathrm{H}_{2} \mathrm{O}\right)_{2}\right]$. $3 \mathrm{H}_{2} \mathrm{O}(\mathbf{1 - R})$ and $\left[\mathrm{Zn}_{4}\left((S)-\mathrm{H}_{3} \mathrm{~L}\right)_{2}(\mathrm{OH})_{2}\left(\mathrm{H}_{2} \mathrm{O}\right)_{2}\right] \cdot 2 \mathrm{H}_{2} \mathrm{O}(\mathbf{1}-\boldsymbol{S})$ were synthesized under hydrothermal conditions. Their crystal structures, thermal stabilities, circular dichroism (CD) spectra, nonlinear optical (NLO) and fluorescent sensing properties were studied.

\section{Experimental section}

General methods and materials

All other reagents and solvents were purchased commercially without further purification. The IR spectra were obtained on 


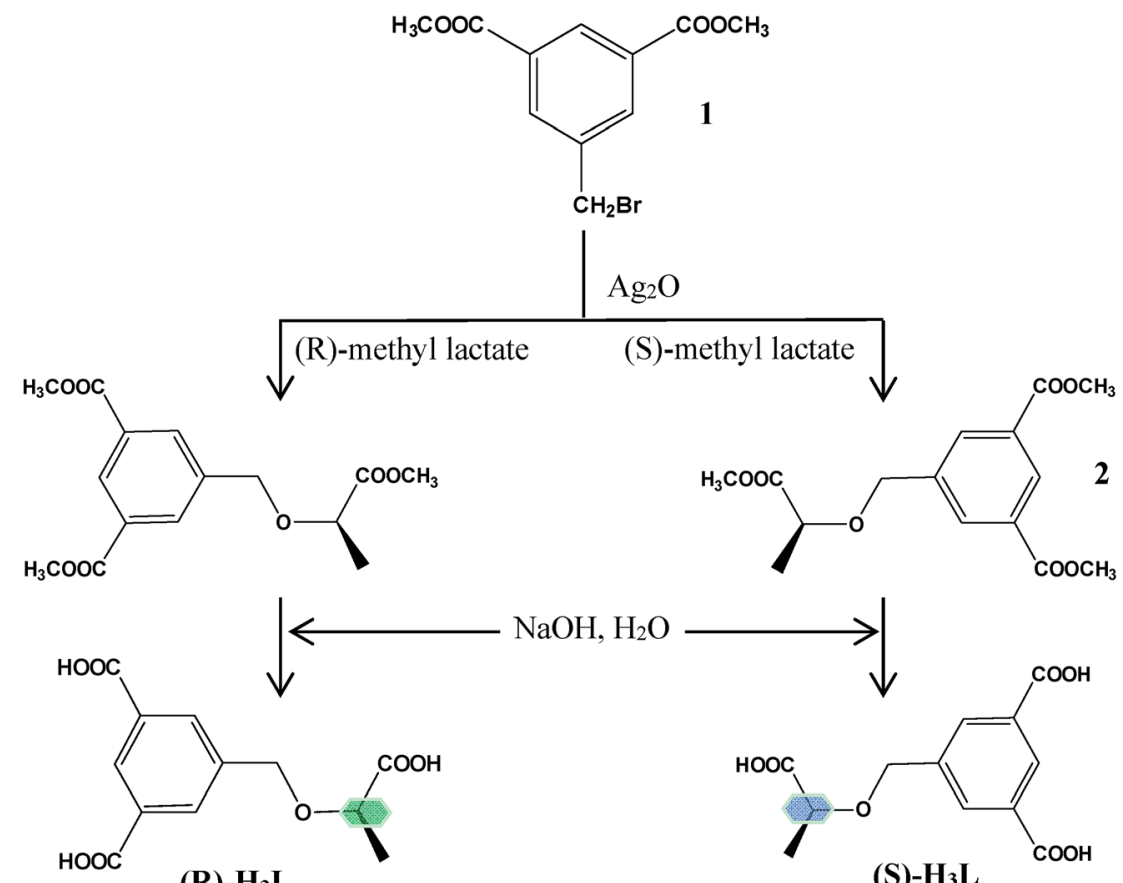

(R)- $\mathbf{H}_{3} \mathbf{L}$

(S)- $\mathbf{H}_{3} \mathrm{~L}$

Scheme 1 Synthesis route of the enantiopure ligands: $(R)-\mathrm{H}_{3} \mathrm{~L}$ and $(S)-\mathrm{H}_{3} \mathrm{~L}$.

a Perkin Elmer Spectrum 100 FT-IR spectrometer equipped with a DGTS detector (32 scans) using $\mathrm{KBr}$ disks in the range $4000-500 \mathrm{~cm}^{-1}$. Elemental analysis for $\mathrm{C}, \mathrm{H}$ were performed on a Perkin-Elmer 240 elemental analyzer. Thermal analyses were performed on a SDT 2960 thermal analyzer from room temperature to $800{ }^{\circ} \mathrm{C}$ at a heating rate of $20^{\circ} \mathrm{C} \mathrm{min}^{-1}$ under nitrogen flow. Powder X-ray diffraction (PXRD) data were collected on a Bruker-D8 Advance X-ray diffractometer with $\mathrm{Cu} \mathrm{K} \alpha$ radiation $(\lambda$ $=1.5406 \AA$ ) and $2 \theta$ ranging from 5 to $50^{\circ}$. Circular dichroism (CD) spectra of solid samples were recorded at room temperature with a MOS-450 spectrometer (KBr pellets). The second-harmonic generation (SHG) intensity was approximately estimated by measuring microcrystalline samples relative to urea. Solid UVvisible spectra were obtained in the $200-800 \mathrm{~nm}$ range on a JASCO UVIDEC-660 spectrophotometer. A pulsed Q-switched Nd:YAG laser at a wavelength of $1064 \mathrm{~nm}$ was used to generate a SHG signal from powder samples. The backscattered SHG light was collected by a spherical concave mirror and passed through a filter that transmits only $532 \mathrm{~nm}$ radiation. ${ }^{6}$ The luminescent spectrum was taken an Edinburgh FLS 920 fluorescence spectrophotometer at room temperature.

Synthesis of the ligand 5-(1-carboxyethoxymethyl)-isophthalic acid $\left(R-H_{3} \mathbf{L}\right.$ and $\left.S-H_{3} \mathbf{L}\right)$. 5-Bromomethyl-isophthalic acid dimethyl ester $(\mathbf{1}, 1.43 \mathrm{~g}, 5 \mathrm{mmol}){ }^{7}(S)$-methyl lactate or $(R)$-methyl lactate $(0.83 \mathrm{~g}, 8 \mathrm{mmol})$ and $\mathrm{Ag}_{2} \mathrm{O}(1.16 \mathrm{~g}, 5 \mathrm{mmol})$ were dissolved in $30 \mathrm{~mL} \mathrm{CH} \mathrm{Cl}_{2}$. The mixture was stirred under $\mathrm{N}_{2}$ atmosphere for $48 \mathrm{~h}$. Then the resulting residue was purified with the method of column chromatography (silica gel eluted by the mixed solvent of $25 \%$ ethyl acetate and petroleum ether). The ester $(2,0.93 \mathrm{~g}, 3$ $\mathrm{mmol})$ and $\mathrm{NaOH}(0.54 \mathrm{~g}, 13.5 \mathrm{mmol})$ were dissolved in a mixed solvent of distilled water $(10 \mathrm{~mL})$ and methanol $(2 \mathrm{~mL})$, being stirred at $55{ }^{\circ} \mathrm{C}$ for $12 \mathrm{~h}$. Concentrated hydrochloric acid was added to the solution until the $\mathrm{pH}$ value of the solution attained to $1-2$, white solid was then produced as the product $\left(R-\mathbf{H}_{3} \mathbf{L}\right.$ and $\boldsymbol{S}$ $\left.\mathbf{H}_{3} \mathbf{L}\right) .{ }^{1} \mathrm{H}$ NMR $(400 \mathrm{MHz}$, DMSO) $\delta=8.39(\mathrm{~s}, 1 \mathrm{H}), 8.13(\mathrm{~s}, 2 \mathrm{H}), 4.67$ (dd, 2H), $4.08(\mathrm{q}, 1 \mathrm{H}), 1.36(\mathrm{~d}, 3 \mathrm{H})$.

Synthesis of $\left\{\left[\mathrm{Zn}_{4}((\mathrm{R})-\mathrm{CIA})_{2}(\mathrm{OH})_{2}\left(\mathrm{H}_{2} \mathrm{O}\right)_{2}\right] \cdot 3 \mathrm{H}_{2} \mathrm{O}\right\}_{n} \quad(1-R)$. $\boldsymbol{R}-\mathrm{H}_{3} \mathbf{L}(0.05 \mathrm{mmol}, 15.5 \mathrm{mg}), \mathrm{ZnCl}_{2} \cdot 6 \mathrm{H}_{2} \mathrm{O}(0.1 \mathrm{mmol}, 24.7 \mathrm{mg})$ and $\mathrm{H}_{2} \mathrm{O}(6 \mathrm{~mL})$ were mixed together, with solid $\mathrm{NaOH}$ added to adjust the $\mathrm{pH}$ value to about 5.5. The mixture was then sealed in a $20 \mathrm{~mL}$ Teflon-lined stainless steel vessel and heated at $130{ }^{\circ} \mathrm{C}$ for 3 days. After cooled to room temperature gradually, colorless transparent crystals were obtained after washed with water (57.1\%, bass on $\mathrm{Zn}$ ). Elemental analysis (\%) calcd for $\mathrm{C}_{24} \mathrm{H}_{29} \mathrm{Zn}_{4} \mathrm{O}_{20.5}$ (906.95): C, 31.78; H, 3.22; found: C, 30.21; H, 3.07. IR (KBr pellet, $\left.\mathrm{cm}^{-1}\right): 3603(\mathrm{w}), 3544(\mathrm{~m}), 3418(\mathrm{~s}), 2988$ (m), $2937(\mathrm{~m}), 1620(\mathrm{~s}), 1562(\mathrm{~s}), 1452(\mathrm{~m}), 1412(\mathrm{~s}), 1361(\mathrm{~m})$, $1123(\mathrm{w}), 1091(\mathrm{~m}), 1067(\mathrm{w}), 1050(\mathrm{w}), 1032(\mathrm{w}), 933(\mathrm{~m}), 908$ $(\mathrm{m}), 781(\mathrm{~s}), 722(\mathrm{~s}), 569(\mathrm{w}), 529(\mathrm{w})$.

Synthesis of $\left\{\left[\mathrm{Zn}_{4}((S) \text {-CIA })_{2}(\mathrm{OH})_{2}\left(\mathrm{H}_{2} \mathrm{O}\right)_{2}\right] \cdot 2 \mathrm{H}_{2} \mathrm{O}\right\}_{n}(1-S)$. The same procedure as that for $\mathbf{1}-\boldsymbol{R}$ was conducted except that $\boldsymbol{S}-\mathbf{H}_{3} \mathbf{L}$ was used. Colorless crystals $(55.2 \%$, bass on $\mathrm{Zn})$ were obtained. Elemental analysis (\%) calcd for $\mathrm{C}_{24} \mathrm{H}_{28} \mathrm{O}_{20} \mathrm{Zn}_{4}$ (897.94): C, 32.09; $\mathrm{H}$, 3.14; found: C, 31.01; $\mathrm{H}, 3.02$. IR (KBr pellet, $\left.\mathrm{cm}^{-1}\right)$ : $3604(\mathrm{w})$, $3546(\mathrm{~m}), 3415(\mathrm{~s}), 2988(\mathrm{~m}), 2938(\mathrm{~m}), 1620(\mathrm{~s}), 1562(\mathrm{~s}), 1453(\mathrm{~m})$, $1415(\mathrm{~s}), 1361(\mathrm{~m}), 1124(\mathrm{w}), 1090(\mathrm{~m}), 1068(\mathrm{w}), 1050(\mathrm{w}), 1033$ (w), $933(\mathrm{~m}), 908(\mathrm{~m}), 781(\mathrm{~s}), 722(\mathrm{~s}), 569(\mathrm{w}), 529(\mathrm{w})$.

$\mathrm{X}$-ray crystallography. Single-crystal X-ray diffraction data for complexes $\mathbf{1 - R}$ and $\mathbf{1 - S}$ were collected. The intensity data of crystals were obtained on a Rigaku R-AXIS RAPID imaging plate diffractometer with graphite-monochromated Mo $\mathrm{K} \alpha(\lambda=$ $0.71073 \AA$ ) at $291 \mathrm{~K}$. Empirical absorption corrections based one 
Table 1 Crystal data and structure refinement for CCPs 1-R and 1-S

\begin{tabular}{|c|c|c|}
\hline & $1-R$ & $1-S$ \\
\hline Empirical formula & $\mathrm{C}_{24} \mathrm{H}_{29} \mathrm{Zn}_{4} \mathrm{O}_{20.5}$ & $\mathrm{C}_{24} \mathrm{H}_{29} \mathrm{Zn}_{4} \mathrm{O}_{20}$ \\
\hline$F_{\mathrm{w}}$ & 906.95 & 897.94 \\
\hline Crystal system & Triclinic & Triclinic \\
\hline Space group & $P 1$ & $P 1$ \\
\hline$a(\AA)$ & $8.1196(5)$ & $8.1097(3)$ \\
\hline$b(\AA)$ & $9.4949(7)$ & $9.5040(4)$ \\
\hline$c(\AA)$ & $10.9553(7)$ & $10.9547(4)$ \\
\hline$\alpha$ (deg) & $105.246(6)$ & $105.0820(10)$ \\
\hline$\beta$ (deg) & $108.647(6)$ & $108.5090(10)$ \\
\hline$\gamma(\mathrm{deg})$ & $95.760(6)$ & $95.9140(10)$ \\
\hline$V\left(\AA^{3}\right)$ & $756.22(9)$ & $757.02(5)$ \\
\hline$Z$ & 1 & 1 \\
\hline$D_{\text {calc }}\left(\mathrm{g} \mathrm{cm}^{-3}\right)$ & 1.992 & 1.970 \\
\hline$\mu\left(\mathrm{mm}^{-1}\right)$ & 3.227 & 3.221 \\
\hline$F(000)$ & 457 & 452 \\
\hline Collected/unique & $5327 / 4333$ & $13709 / 5278$ \\
\hline$R$ (int) & 0.0198 & 0.0299 \\
\hline GOF on $F^{2}$ & 1.005 & 1.018 \\
\hline$R_{1}^{a}[I>2 \sigma(I)]$ & 0.0283 & 0.0267 \\
\hline $\mathrm{w} R_{2}^{b}[I>\sigma(I)]$ & 0.0577 & 0.0666 \\
\hline$R_{1}{ }^{a}($ all $)$ & 0.0359 & 0.0379 \\
\hline $\mathrm{w} R_{2}^{b}$ (all) & 0.0605 & 0.0718 \\
\hline Flack parameters & $0.04(2)$ & $-0.01(2)$ \\
\hline
\end{tabular}

equivalent reflections were applied. The structures were solved by direct methods and refined by full-matrix least-squares methods on $F^{2}$ using SHELXS-97 crystallographic software package. ${ }^{8}$ All isolated $\mathrm{O}$ atoms have been considered as water atoms. The crystal parameters, data collection, and refinement results for 1-R and 1-S are summarized in Table 1. Crystallography data have been deposited to the Cambridge Crystallography Data Centre with deposition numbers CCDC no. 1565357 and 1565358 for $\mathbf{1}-\boldsymbol{R}$ and $\mathbf{1}-\boldsymbol{S}$.

\section{Results and discussion}

\section{Structural description}

CCPs 1- $\boldsymbol{R}$ and 1-S crystallized in a same chiral space group $P 1$ with a Flack parameter of 0.09(4) and single-crystal XRD analyses revealed that the two compounds are isostructural, thus only the structure of $\mathbf{1 - R}$ is described in detail. In an asymmetric unit, there are four crystallographically unique $\mathrm{Zn}$ (II) atoms, two deprotonated $\boldsymbol{R}-\mathbf{H}_{3} \mathbf{L}^{3-}$ ligands, two coordinated water molecules, two $\mu_{3}$-bridging hydroxyl anions and three guest water molecules. The chiral carbon in CCP 1- $R$ retains the same $R$ configuration as that in the starting material of $(\boldsymbol{R})-\mathbf{H}_{3} \mathbf{L}$. The $\mathrm{Zn} 1$ ion is 6-coordinated in a distorted octahedron defined by three oxygen atoms from three carboxylate groups from three $\boldsymbol{R}$ $\mathbf{H}_{3} \mathbf{L}^{3-}$ ligands, one oxygen atom from ether group of $R-\mathrm{H}_{3} \mathrm{~L}^{3-}$ ligand and two $\mu_{3}$-bridging hydroxyl anions. The $\mathrm{Zn} 2$ ion is also in a 6-coordinated distorted octahedron by four oxygen atoms from four carboxylate groups of $\boldsymbol{R}-\mathbf{H}_{3} \mathbf{L}^{3-}$ ligands, one $\mu_{3}$ bridging hydroxyl anion and one guest water molecule. The coordination forms of $\mathrm{Zn} 3$ and $\mathrm{Zn} 4$ ion are same as those of $\mathrm{Zn} 1$ and $\mathrm{Zn} 2$, respectively (Fig. 1(a)). The $\mathrm{Zn}-\mathrm{O}$ bond lengths range from 1.975(6) to 2.337(7) $\AA$ and the $\mathrm{O}-\mathrm{Zn}-\mathrm{O}$ bond angles range from $70.8(3)$ to $179.4(3)$, which are all in the normal ranges (Table S1†).

There is an infinite chiral chain in $\mathbf{1}-\boldsymbol{R}$ and $\mathbf{1 - S}$, which is constructed by $\mathrm{Zn}$ ions linking carboxylate groups of $(\boldsymbol{R})-\mathbf{H}_{3} \mathbf{L}$ or

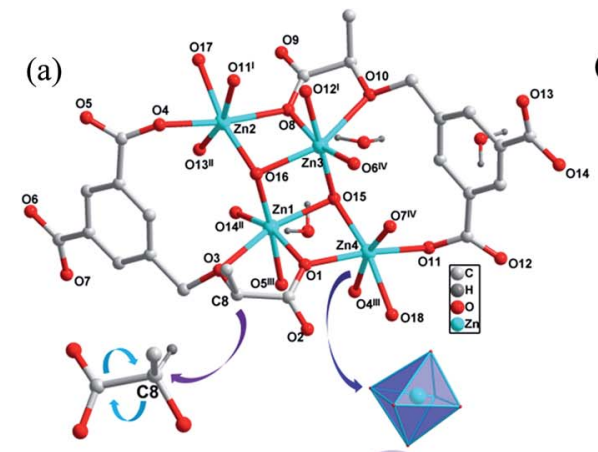

(c)

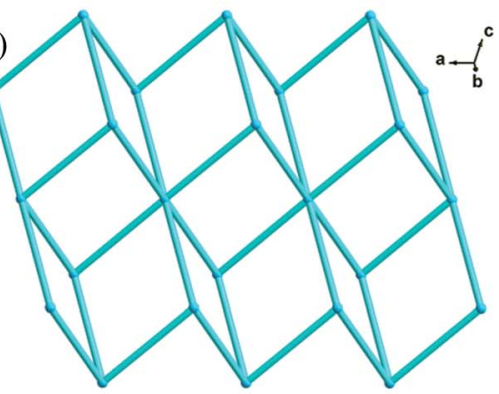

(b)
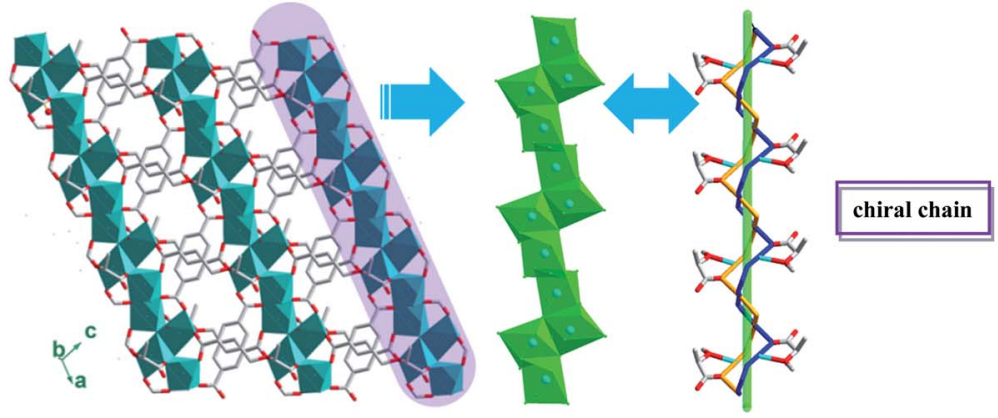

Fig. 1 (a) Stick-ball representation of the asymmetric unit of CCP 1- $R$. (b) 2D framework of 1- $R$ constructed from chiral chains. (c) Schematic view of $2 \mathrm{D}(3,6)$-connected framework with a point symbol of $\left(4^{3}\right)_{2}\left(4^{6} \cdot 6^{6} \cdot 8^{3}\right)$. Symmetry codes: (I) $-1+x, y, z ;$ (II) $-1+x, y, 1+z ;$ (III) $1+x, y, z ;$ (IV) $1+x, y, 1+z$. 
(S)- $\mathbf{H}_{3} \mathbf{L}$ ligands and $\mu_{3}$-bridging hydroxyl anions. In one [Zncarboxylate groups] helical tube, there are $R$ direction [Zn2O9-Zn3-O6-C12-O7-Zn4-O4] fragment, with the $\mathrm{Zn}-\mathrm{Zn}$ distance between each fragment of $3.5337 \AA$ and the pitch of the helix of $8.12 \AA$. Another S direction [Zn2-O13-C20-O14-Zn1O1-Zn4-O11] fragment shares the same pitch of the helix as $R$ direction fragment, with the chiral carbons of $(\boldsymbol{R})-\mathbf{H}_{3} \mathbf{L}$ ligands keeping the original direction. Interestingly, the $\mathrm{Zn}$ ions are closely connected by $\mu_{3}$-bridging hydroxyl anions, which are indispensable during the construction of chiral chains. The experimental results also confirmed this inference, as it was found that the CCPs could not be generated without the presence of $\mathrm{NaOH}$. Furthermore, the chiral chains connected with each other by the $(\boldsymbol{R})-\mathbf{H}_{3} \mathbf{L}$ ligands and expanded indefinitely, resulting in a $2 \mathrm{D}$ network structure(Fig. 1(b)). In order to further analyze and understand this $2 \mathrm{D}$ structure of $\mathbf{1}-\boldsymbol{R}$, topological analysis is carried out. If the $(\boldsymbol{R})-\mathbf{H}_{3} \mathbf{L}^{3-}$ ligands are considered as 3-connected node and $\mathrm{Zn}$ ions are considered as 6-connected notes, the 2D structure of CCP 1- $R$ can be regarded as a 3,6-c net and kgd topological type with a point symbol of $\left(4^{3}\right)_{2}\left(4^{6} \cdot 6^{6} \cdot 8^{3}\right)$ (Fig. 1(c)).

\section{PXRD patterns and thermal stability analysis}

For further confirming the phase purity of the crystalline, the experimental and simulated PXRD patterns of $\mathbf{1}-\boldsymbol{R}$ and $\mathbf{1 - S}$ are compared (Fig. S1†). The experimental PXRD patterns corroborate the simulated patterns generated from single-crystal diffraction data, indicating the purity of the crustal samples. The differences in intensity may be due to the preferred orientation of the powder samples. When studying the stability of the CCPs, the existence of bulk water in $\mathbf{1}-\boldsymbol{R}$ and $\mathbf{1}-\boldsymbol{S}$ is also observed by TGA under air atmosphere (Fig. S2 $\dagger$ ). The weight loss process for 1-R can be divided into two steps. The first weight loss of $9.30 \%$ occurring at $50-270{ }^{\circ} \mathrm{C}$ corresponds to the total loss of the five water molecules (calcd 9.93\%). Then the weight loss of $57.96 \%$ occurring at $270-690{ }^{\circ} \mathrm{C}$ could be ascribed to the total loss of the two ligand molecules (calcd 59.16). The remaining weight of $32.74 \%$ corresponds to the formation of $\mathrm{ZnO}$. The weight loss process for 1-S is similar to that of $\mathbf{1 - R}$ (Fig. S3†).

\section{The solid-state circular dichroism (CD) spectra analysis}

In order to further demonstrate the homochirality of CCPs 1-R and 1-S, solid-state circular dichroism (CD) analysis has been conducted. As shown in Fig. 2, the CD spectrum for the bulk sample of $\mathbf{1 - R}$ exhibits a positive Cotton effect with peak at $332 \mathrm{~nm}$, while the bulk sample of $\mathbf{1 - S}$ exhibits negative Cotton effect at the same wavelength. The result confirms that the CCPs $\mathbf{1}-\boldsymbol{R}$ and $\mathbf{1 - S}$ are homochiral enatiomers.

\section{Second-harmonic generation (SHG) properties}

Nonlinear optical (NLO) active materials have been widely studied and applied in laser frequency conversion and electrooptical devices at present. As one of the most common NLO behaviors, second-harmonic generation (SHG) have been of increasing interest to synthetic chemists. ${ }^{9}$ SHG active materials has found the most common use in the laser industry.

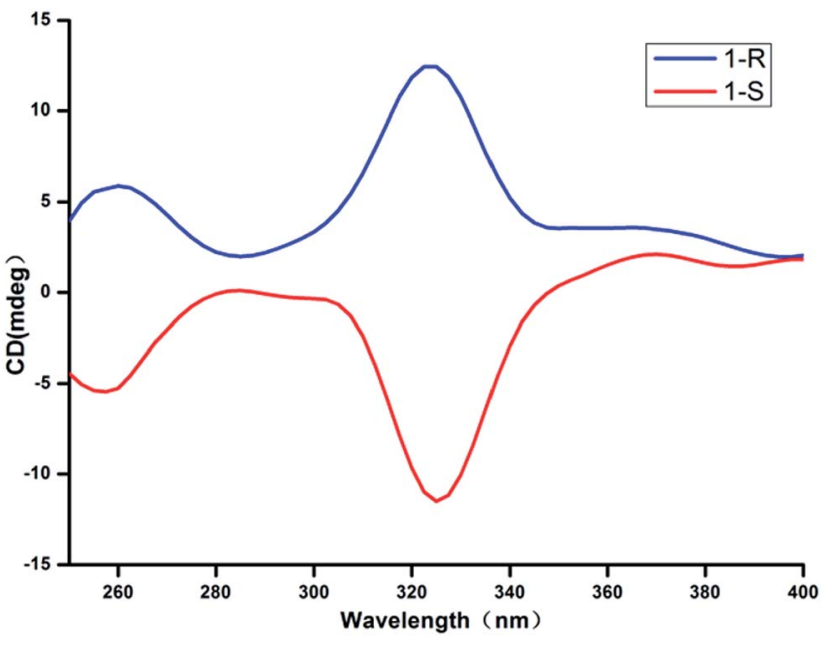

Fig. 2 Solid-state CD spectra of CCPs 1-R and 1-S.

Coordination polymers have been regarded as an attractive category of SHG active materials, as the structures of which could be fine tuned on molecular level. The rational design and synthesis of CCPs in a systematic way might give rise to potential SHG active materials. Supramolecular aggregates and chirality could significantly enhance the nonlinear optical properties. ${ }^{10}$ And $\mathrm{d}^{10}$ metal, such as $\mathrm{Zn}$ and $\mathrm{Cd}$ are most adopted for the construction of coordination polymers with SHG activities. ${ }^{11}$ Due to the fact that the pair of CCPs $1-R$ and $1-S$ are crystallized in the non-centrosymmetric chiral space groups and constructed from metal Zn, their SHG activities are investigated. Their SHG properties have been carried out by the Kurtz-Perry method using a powder technique. ${ }^{12}$ Preliminary experimental results show that $\mathbf{1} \boldsymbol{R}$ and $\mathbf{1}-\boldsymbol{S}$ are all 0.3 times as much as that of urea. CCPs 1-R and 1-S exhibit lower SHG response than that of urea, which might be resulted from the lacking of a donor-acceptor system in final structures.

\section{Fluorescent selective recognition}

It was ever reported that the fluorescence of some coordination polymers could be quenched by nitroaromatic compounds under certain conditions, suggesting their potential applications for recognizing nitroaromatic explosives. ${ }^{13}$ In our investigation, trinitrotoluene (TNT) are used to investigate the luminescent quenching effect of CCPs 1-R and 1-S. The pair of enatiomers $\mathbf{1}-\boldsymbol{R}$ and $\mathbf{1}-\boldsymbol{S}$ show very similar results.

The sample of CCP $\mathbf{1}-\boldsymbol{R}$ was dispersed in DMF solution so that the TNT molecules could adhere to the particle surface of CCP, facilitating possible host-guest interactions. As shown in Fig. 3, with the concentration of TNT gradually increased, the luminescence intensity of CCP $\mathbf{1} \boldsymbol{R}$ decreased significantly. According to the previous reports, such luminescence quenching might be related to the donor-acceptor electron-transfer mechanism. The electrons transfers from an excited CCP 1-R to the electron deficient TNT, resulting in the occurrence of fluorescence quenching of CCP 1-R. ${ }^{14}$

According to some reports, not only nitro aromatic compounds but also metal cations could have an quenching 
(a)

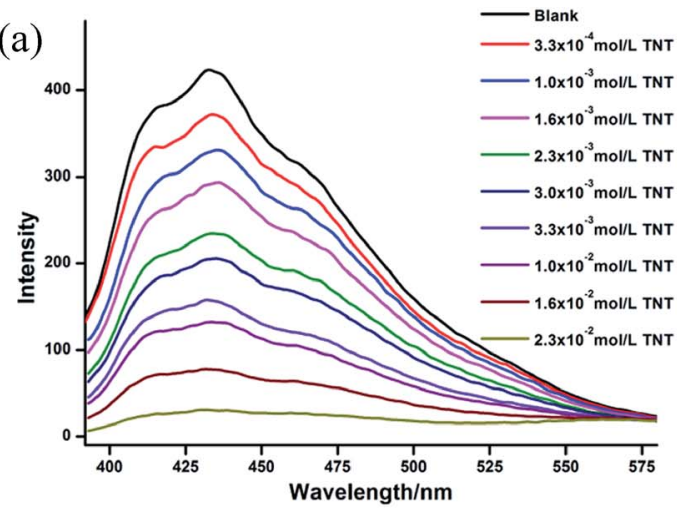

(b)

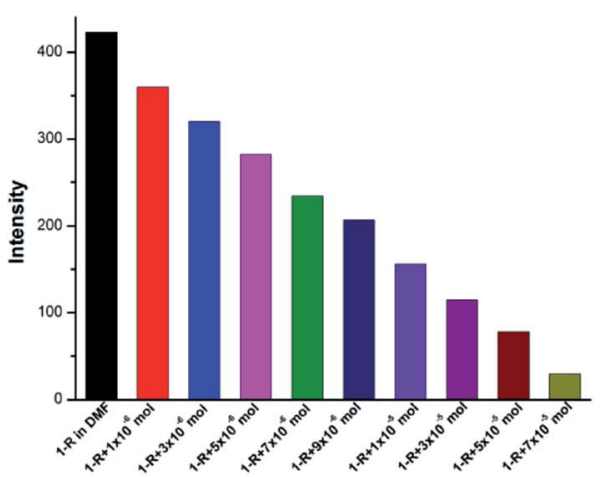

Fig. 3 (a) The fluorescent spectra of 1-R in DMF with different amount of TNT. (b) Luminescent intensity diversity at $441 \mathrm{~nm}$ of 1-R in DMF with different amount of TNT.

(a)

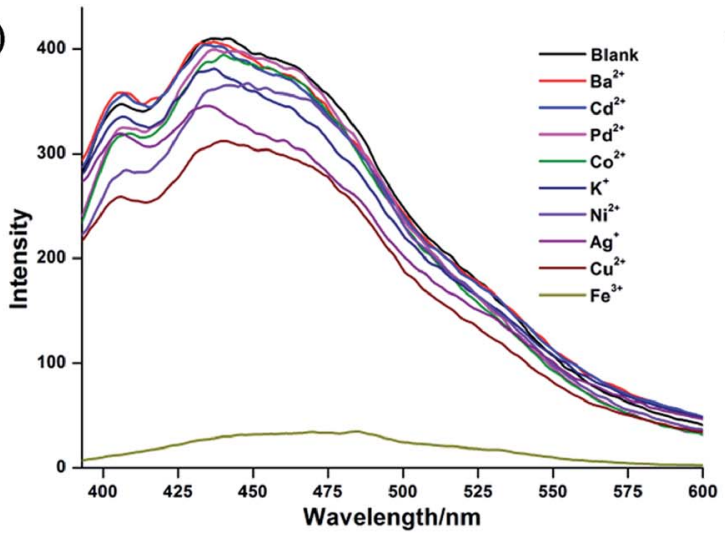

(b)

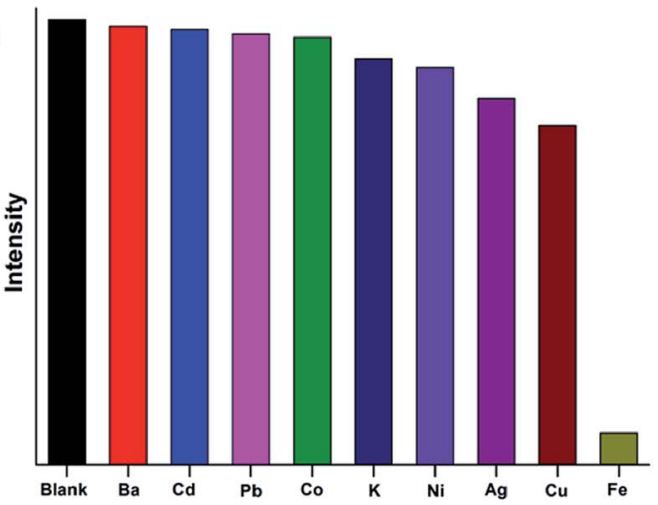

Fig. 4 (a) Luminescent spectra of $1-S$ in the presence of metal cations of $1 \times 10^{-3} \mathrm{~mol} \mathrm{~L}^{-1}$. (b) Luminescent intensity diversity at $441 \mathrm{~nm}$ of $1-\mathrm{S}$ when treated with various cations of $1 \times 10^{-3} \mathrm{~mol} \mathrm{~L}^{-1}$.

effect toward some coordination polymers. ${ }^{15}$ In order to further study the fluorescent selective recognition of CCPs, different metal cations are screened to investigate their effect on the fluorescence of CCPs 1- $\boldsymbol{R}$ and 1-S. As a result, CCPs 1-R and 1-S also show very similar selectivity toward the metal cations.
CCP 1-S was added in DMF and stirred for $24 \mathrm{~h}$, so that 1-S was sufficiently dispersed in DMF solution. For the purpose of verifying the selectivity of CCP-1 toward the metal cations, nine metal nitrates, $\mathrm{M}\left(\mathrm{NO}_{3}\right)_{x}\left(\mathrm{M}=\mathrm{Ba}^{2+}, \mathrm{Cd}^{2+}, \mathrm{Pb}^{2+}, \mathrm{Co}^{2+}, \mathrm{K}^{+}, \mathrm{Ni}^{2+}\right.$, $\left.\mathrm{Ag}^{+}, \mathrm{Cu}^{2+}, \mathrm{Fe}^{3+}\right)$ are used for screening. The aqueous solution (a)

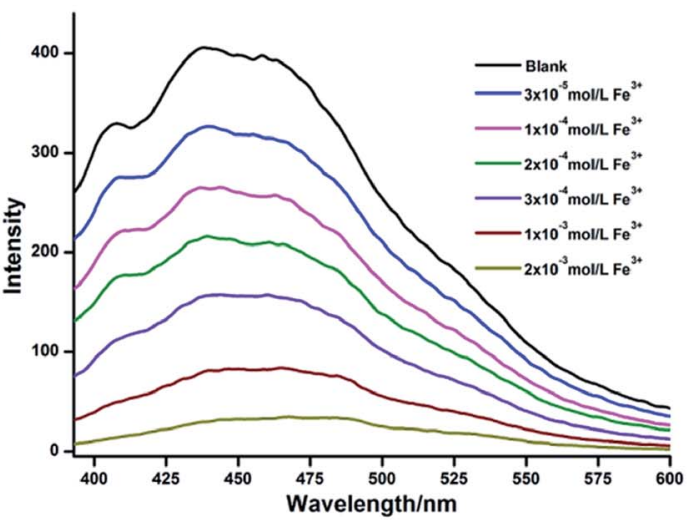

(b)

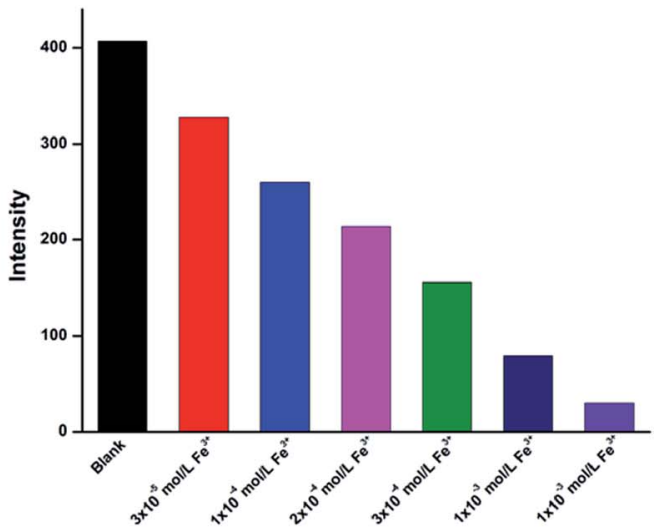

Fig. 5 (a) The fluorescent spectra of 1-S in DMF with different amount of Fe $\mathrm{F}^{3+}$. (b) Luminescent intensity diversity at $441 \mathrm{~nm}$ of $1-\mathrm{S}$ in DMF with different amount of $\mathrm{Fe}^{3+}$. 
containing $1 \times 10^{-3} \mathrm{~mol} \mathrm{~L}^{-1} \mathrm{M}\left(\mathrm{NO}_{3}\right)_{x}$ was added to the suspension of CCP 1-S, respectively. The results are shown in Fig. 4. It was found that $\mathrm{Fe}^{3+}$ have the most obviously inhibiting effect on the luminescence of CCP 1-S when adding the same molar amount of various metal cations. The results indicate that CCP 1-S has an obvious selection effect toward $\mathrm{Fe}^{3+}$.

As shown in Fig. 5, as we increase the amount of $\mathrm{Fe}^{3+}$, the luminescence intensity of CCP 1-S decreased obviously. In previous reports, the mechanisms for luminescence quenching effect of $\mathrm{Fe}^{3+}$ ion were proposed as the collapse of the framework, cationic exchange, and the physical interactions between $\mathrm{Fe}^{3+}$ ion and carboxylic acid. ${ }^{16}$ To further probe the possible luminescence quenching mechanism of $\mathrm{Fe}^{3+}$ ion, we removed the $\mathrm{Fe}^{3+}$ ion from the suspension of CCP 1-S by filtrating and washing it with water and ethanol for several times. The resulted sample was used for powder XRD and luminescence analysis. The results show that the framework of CCP 1-S was retained the same as the original and the luminescence also returned to normal. We inferred that the luminescence quenching mechanism of $\mathrm{Fe}^{3+}$ ion toward CCP 1-S might be ascribed to the physical interactions between $\mathrm{Fe}^{3+}$ ion and carboxylic group of CCP 1-S. This also indicates that CCP 1-S could be recycled in metal recognition.

\section{Conclusion}

In summary, we synthesized a pair of novel Zn(II) enantiomeric CCPs based on a pair of chiral multicarboxylate ligand, $(R)$ - or (S)-5-(1-carboxyethoxymethyl)-isophthalic acid. The two enantiomeric CCPs $\mathbf{1}-\boldsymbol{R}$ and $\mathbf{1}-\boldsymbol{S}$ are isostructural and also exhibit very similar functional properties. The SHG optical results show that CCPs 1- $\boldsymbol{R}$ and 1-S have medium SHG activities which could be used as potential optical materials. Furthermore, the luminescence of CCP 1 could be effectively quenched by trinitrotoluene and $\mathrm{Fe}^{3+}$, indicating their potential application as sensory materials. At the same time, it was also found that the luminescence of CCP 1 could return to normal after the $\mathrm{Fe}^{3+}$ ion being completely removed from CCP 1 , which indicating that CCP 1 could be recycled and used in metal recognition.

\section{Conflicts of interest}

There are no conflicts to declare.

\section{Acknowledgements}

This work is financially supported by the National Natural Science Foundation of China (No. 21371052 \& 21272061).

\section{References}

1 (a) R. Hoffmann, The Same and Not the Same, Columbia University Press, New York, 1995; (b) G. Keliang and W. Dongzhi, Appl. Microbiol. Biotechnol., 2006, 70, 135139; (c) S. Sharma, M. Chauhan, A. Jamsheera, S. Tabassum and F. Arjmand, Inorg. Chim. Acta, 2017, 458, 8-27; (d) M. Liu, L. Zhang and T. Wang, Chem. Rev.,
2015, 115, 7304-7397; (e) J.-Z. Gu, Y.-H. Cui, J. Wu and A. M. Kirillov, $R S C A d v ., 2015$, 5, 78889-78901; $(f)$ N. Chen, M.-X. Li, P. Yang, X. He, M. Shao and S.-R. Zhu, Cryst. Growth Des., 2013, 13, 2650-2660; (g) M. Yadav, A. Bhunia, S. K. Jana and P. W. Roesky, Inorg. Chem., 2016, 55, 2701-2708.

2 (a) D. W. Lim, J. W. Yoon, K. Y. Ryu and M. P. Suh, Angew. Chem., Int. Ed., 2012, 51, 9952; (b) C. Wang, T. Zhang and W.-B. Lin, Chem. Rev., 2012, 112, 1084; (c) J. Zhang and X.-H. Bu, Angew. Chem., Int. Ed., 2007, 119, 6227; (d) J. Crassous, Chem. Soc. Rev., 2009, 38, 830; (e) T. Hang, D.-W. Fu, Q. Ye and R.-G. Xiong, Cryst. Growth Des., 2009, 9, 2026; (f) X.-L. Zhao, D. Tian, Q. Gao, H.-W. Sun, J. Xu and X.-H. Bu, Dalton Trans., 2016, 45, 1040.

3 (a) G. Echue, I. Hamley, G. C. Lloyd Jones and C. F. Faul, Langmuir, 2016, 32, 9023-9032; (b) D.-W. Gao, Q. Gu and S.-L. You, ACS Catal., 2014, 4, 2741-2745; (c) S. Yoshioka, Y. Inokuma, M. Hoshino, T. Sato and M. Fujita, Chem. Sci., 2015, 6, 3765-3768; (d) L. Zhang, T. Wang, Z. Shen and M. Liu, Adv. Mater., 2016, 28, 1044-1059; (e) D. N. Dybtsev, M. P. Yutkin, E. V. Peresypkina, A. V. Virovets, C. Serre, G. Férey and V. P. Fedin, Inorg. Chem., 2007, 46, 6843; (f) J. Zhang and X.-H. Bu, Chem. Commun., 2008, 1756; (g) J. Zhang and X.-H. Bu, Angew. Chem., Int. Ed., 2007, 46, 6115; (h) J. J. Bodwin, A. D. Cutland, R. G. Malkani and V. L. Pecoraro, Coord. Chem. Rev., 2001, 216-217, 489-512.

4 (a) M. Jung, H. Kim, K. Baek and K. Kim, Angew. Chem., Int. Ed., 2008, 47, 5755; (b) L.-H. Cao, Y.-L. Wei, Y. Yang, H. Xu, S.-Q. Zang, H.-W. Hou and T. C. W. Mak, Cryst. Growth Des., 2014, 14, 1827-1838; (c) Z.-J. Lin, J. Lu, M. Hong and R. Cao, Chem. Soc. Rev., 2014, 43, 5867-5895; (d) L.-B. Sun, J.-R. Li, W.-G. Lu, Z.-Y. Gu, Z.-P. Luo and H.-C. Zhou, J. Am. Chem. Soc., 2012, 134, 15923; (e) W.-J. Zhuang, D.-Q. Yuan, D.-H. Liu, C.-L. Zhong, J.-R. Li and H.-C. Zhou, Chem. Mater., 2012, 24, 18; $(f)$ H. Furukawa, J. Kim, K. E. Plass and O. M. Yaghi, J. Am. Chem. Soc., 2006, 128, 8398.

5 (a) H.-T. Ye, C.-Y. Ren, G.-F. Hou, Y.-H. Yu, X. Xu, J.-S. Gao, P.-F. Yan and S.-W. Ng, Cryst. Growth Des., 2014, 14, 33093318; (b) Y.-H. Yu, H.-T. Ye, G.-F. Hou, C.-Y. Ren, J.-S. Gao and P.-F. Yan, Cryst. Growth Des., 2016, 16, 5669-5677; (c) T. Liu, Y.-H. Yu, H.-Z. Zhang, W.-H. Jiang, J.-S. Gao and G.-F. Hou, Cryst. Growth Des., 2017, 17, 1788-1795; (d) J.-H. Huang, G.-F. Hou, D.-S. Ma, Y.-H. Yu, W.-H. Jiang, Q. Huang and J.-S. Gao, RSC Adv., 2017, 7, 18650-18657.

6 R.-G. Xiong, X. Xue, H. Zhao, X.-Z. You, B. F. Abrahams and Z.-L. Xue, Angew. Chem., Int. Ed., 2002, 41, 3800.

7 (a) J. Xu, R. Wang, Y. Li, Z. Gao, R. Yao, S. Wang and B. Wu, Eur. J. Inorg. Chem., 2012, 3349-3360; (b) T. Routasalo, J. Helaja, J. Kavakka and A. M. P. Koskinen, Eur. J. Org. Chem., 2008, 3190.

8 G. M. Sheldrick, Acta Crystallogr., Sect. A: Found. Crystallogr., 2008, 64, 112.

9 (a) E. Cariati, G. Cavallo, A. Forni, G. Leem, P. Metrangolo, F. Meyer, T. Pilati, G. Resnati, S. Righetto, G. Terraneo and E. Tordin, Cryst. Growth Des., 2011, 11, 5642-5648; (b) S. Rosenne, E. Grinvald, E. Shirman, L. Neeman, S. Dutta, 
O. Bar-Elli, R. Ben-Zvi, E. Oksenberg, P. Milko, V. Kalchenko, H. Weissman, D. Oron and B. Rybtchinski, Nano Lett., 2015, 15, 7232-7237.

10 (a) T. Verbiest, J. Mater. Chem., 1999, 9, 2005-2012; (b) T. Verbiest, Science, 1998, 282, 913-915.

11 (a) S.-Q. Zang, Y. Su, Y.-Z. Li and Z.-P. Ni, Inorg. Chem., 2006, 45, 174-180; (b) S.-L. Zheng, J.-H. Yang, X.-L. Yu, X.-M. Chen and W.-T. Wong, Inorg. Chem., 2004, 43, 830-838.

12 (a) N. Nwaji, J. Mack, J. Britton and T. Nyokong, New J. Chem., 2017, 41, 2020-2028; (b) S. Kujala, A. Mannila, L. Karvonen, K. Kieu and Z. Sun, Sci. Rep., 2016, 6, 223.

13 (a) S. Pramanik, C. Zheng, X. Zhang, T. J. Emge and J. Li, J. Am. Chem. Soc., 2011, 133, 4153-4155; (b) C. Zhang, Y. Yan, L. Sun, Z. Liang and J. Li, CrystEngComm, 2016, 18, 41024108; (c) M. E. Germain and M. J. Knapp, Chem. Soc. Rev., 2009, 38, 2543-2555.
14 (a) Q. Zheng, F. Yang, M. Deng, Y. Ling, X. Liu, Z. Chen, Y. Wang, L. Weng and Y. Zhou, Inorg. Chem., 2013, 52, 10368; (b) B. Gole, A. K. Bar and P. S. Mukherjee, Chem. Commun., 2011, 47, 12137-12139; (c) G. Y. Wang, L. L. Yang, Y. Li, H. Song, W. J. Ruan, Z. Chang and X. H. Bu, Dalton Trans., 2013, 42, 12865-12868.

15 (a) Q. Tang, S. Liu, Y. Liu, J. Miao, S. Li, L. Zhang, Z. Shi and Z. Zheng, Inorg. Chem., 2013, 52, 2799-2801; (b) Y. J. Cui, Y. F. Yue, G. D. Qian and B. L. Chen, Luminescent functional metal-organic frameworks, Chem. Rev., 2012, 112, 1126-1162.

16 (a) M. Zheng, H. Tan, Z. Xie, L. Zhang, X. Jing and Z. Sun, ACS Appl. Mater. Interfaces, 2013, 5, 1078-1083; (b) G. P. Li, G. Liu, Y. Z. Li, L. Hou, Y. Y. Wang and Z. Zhu, Inorg. Chem., 2016, 55, 3952-3959; (c) M.-M. Chen, X. Zhou, H.-X. Li, X.-X. Yang and J.-P. Lang, Cryst. Growth Des., 2015, 15, 2753-2760. 